\title{
A Bayesian Network Framework for Reject Inference
}

\author{
Andrew Smith \\ Department of Computer Science \\ University of California, San Diego \\ La Jolla, CA 92307 \\ atsmith@cs.ucsd.edu
}

\author{
Charles Elkan \\ Department of Computer Science \\ University of California, San Diego \\ La Jolla, CA 92307 \\ elkan@cs.ucsd.edu
}

\begin{abstract}
Most learning methods assume that the training set is drawn randomly from the population to which the learned model is to be applied. However in many applications this assumption is invalid. For example, lending institutions create models of who is likely to repay a loan from training sets consisting of people in their records to whom loans were given in the past; however, the institution approved loan applications previously based on who was thought unlikely to default. Learning from only approved loans yields an incorrect model because the training set is a biased sample of the general population of applicants. The issue of including rejected samples in the learning process, or alternatively using rejected samples to adjust a model learned from accepted samples only, is called reject inference.

The main contribution of this paper is a systematic analysis of different cases that arise in reject inference, with explanations of which cases arise in various real-world situations. We use Bayesian networks to formalize each case as a set of conditional independence relationships and identify eight cases, including the familiar missing completely at random (MCAR), missing at random (MAR), and missing not at random (MNAR) cases. For each case we present an overview of available learning algorithms. These algorithms have been published in separate fields of research, including epidemiology, econometrics, clinical trial evaluation, sociology, and credit scoring; our second major contribution is to describe these algorithms in a common framework.
\end{abstract}

\section{Categories and Subject Descriptors}

G.3 [Probability and Statistics]: statistical computing; H.2.8 [Database Management]: Database Applicationsdata mining; I.2.6 [Artificial Intelligence]: Learningparameter learning; I.5.2 [Information Storage and Retrieval]: Design Methodology_classifier design and evaluation

Permission to make digital or hard copies of all or part of this work for personal or classroom use is granted without fee provided that copies are not made or distributed for profit or commercial advantage and that copies bear this notice and the full citation on the first page. To copy otherwise, to republish, to post on servers or to redistribute to lists, requires prior specific permission and/or a fee.

KDD'04, August 22-25, 2004, Seattle, Washington, USA.

Copyright 2004 ACM 1-58113-888-1/04/0008 ...\$5.00.

\section{General Terms}

Algorithms, Economics, Human Factors, Theory.

\section{Keywords}

Reject inference, sample selection bias, Heckman estimator, propensity scores, expectation-maximization, Bayesian networks.

\section{THE REJECT INFERENCE PROBLEM}

In the typical reject inference application, the aspect of the data that is to be learned (called the outcome) cannot be observed in some of the samples, called the rejects. These rejects are usually not randomly drawn from the training set, but are related in some way to the outcome. Therefore, if a model is learned from only the data with observable outcomes (the accepts), the model will have been trained on samples with biased selection (as opposed to random selection), since the accepts constitute a skewed sample of the general population. The reject inference problem is to include the rejected data in the learning process to avoid this sample selection bias.

One common instance of reject inference arises in the problem of loan application approval. When people apply for a loan, their application is either accepted or rejected, depending on the lender's guess as to how likely the applicant is to repay the loan. Then the people whose applications were accepted either eventually repay the loan or default on the loan (the outcome). We would like to use a mathematical model to predict how likely a person is to repay the loan, so we can better decide whom to reject or accept, by using a database created by a financial institution; however such databases only have repay/default behavior recorded for the people whose applications were accepted, since the rejected people never had a chance to repay or default on the loan. The accepts clearly constitute a biased sample of all the applicants, so reject inference should help us develop an unbiased model.

Another common example is that of medical treatment. We would like to develop a mathematical model for a particular treatment that predicts the extent to which it will help the patient, measured perhaps by the expected lifetime increase. To create such a model, we would use a database describing many patients and their responses to the treatment. Any realistic database, however, will be biased by sample selection, since it only contains patients whom doctors recommended for the treatment; certainly whether or not a doctor recommends someone for a particular treat- 
ment is related to how much that treatment is expected to benefit the patient. Reject inference should be used to create an unbiased model of how well each patient is likely to respond to the treatment.

So-called active learning is the situation where a learning agent chooses which training examples should be labeled, instead of using a randomly sampled training set [7]. Typically the agent is given a set of unlabeled examples and repeatedly requests labels for the members of this set for which labels are likely to be most informative. Sample selection bias arises in the context of active learning in two ways. Most obviously, a training set chosen via active learning is not a random sample from the whole population. Less obviously, sample selection bias is also an issue when measuring the performance (e.g. the accuracy) of a classifier acquired via active learning. If obtaining labels for examples is expensive, then we will not have the luxury of having a large randomly chosen evaluation set of examples with which to measure performance. We will need to obtain somehow unbiased performance estimates from biased evaluation sets.

Reject inference problems can have binary outcome variables, as in the loan application example, or be regression problems, as in the medical example. Rejection/selection is usually binary, but in some applications it can be multivalued. For example, we can model whether or not to make a particular investment (a binary selection choice), or how much money to invest (a multi-valued choice).

\section{BAYESIAN NETWORKS}

An important part of reject inference lies in the assumptions about how the selection mechanism and the outcome mechanism are related. Bayesian networks provide a natural tool for representing possible relationships, both because they have intuitive causal interpretations, and because they represent knowledge about their variables in a precise way, in the form of conditional independence relationships. Bayesian networks cannot encode that the outcome is observable only when a sample has been selected, but they can encode the conditional independence relationships between selection and outcome.

A Bayesian network is a graphical way to represent how a joint distribution of random variables can be factored. In general any joint probability distribution may be factored in the following way:

$$
\begin{aligned}
& p\left(a_{1}, a_{2}, a_{3}, \ldots, a_{n}\right) \\
& \quad=p\left(a_{1}\right) p\left(a_{2} \mid a_{1}\right) p\left(a_{3} \mid a_{1}, a_{2}\right) \ldots p\left(a_{n} \mid a_{1}, a_{2}, a_{3}, \ldots, a_{n-1}\right) .
\end{aligned}
$$

To represent such a distribution over binary variables requires $2^{n}-1$ parameters; however, Bayesian networks encode information about which variables are conditionally independent, leading to a simpler factoring of the joint distribution, and therefore simpler mathematical models. For example, consider the following Bayesian network:

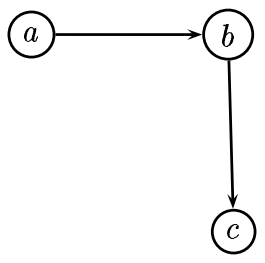

This network encodes the fact that $b$ depends only on $a$ and $c$ depends only on $b$. In other words, $a$ and $c$ are conditionally independent given $b$. The general factoring of the joint distribution can now be simplified because $p(c \mid a, b)=p(c \mid b)$ :

$$
p(a, b, c)=p(a) p(b \mid a) p(c \mid a, b)=p(a) p(b \mid a) p(c \mid b) .
$$

In the case that $a, b$, and $c$ are binary, representing the joint distribution factored in this form can be accomplished with five parameters instead of seven. This reduction in the number of model parameters is possible whenever the situation is described by a Bayesian network whose skeleton (i.e. the graph with the edge directions removed) is not a complete graph. The following Bayesian network has a skeleton that is a complete graph:

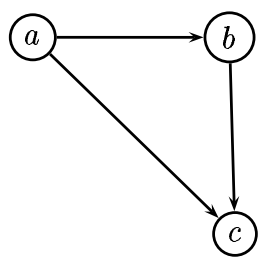

The joint distribution factoring implied by this graph is $p(a, b, c)=p(a) p(b \mid a) p(c \mid a, b)$; however this factoring is true of any distribution over three variables, without loss of generality. This generality can conflict with intuitions about Bayesian networks. Because the edges on the network are directed a particular way we might expect the network to be able to model only particular distributions (i.e. distributions arising from circumstances with the same causal relationships as the network implies), whereas the factoring shows that this network is completely general, for all distributions over three variables [10].

Bayesian networks are good tools for organizing sets of conditional independence relationships because those sets correspond to properties of graphs over which the joint distribution factors. Two variables, $A$ and $B$, in a probability distribution are conditionally independent given a set of other variables, $X$, if information cannot flow between their corresponding vertices in the Bayesian network describing that distribution. For information to flow between $A$ and $B$, there must be an "active path" between them. Clearly there must be a series of edges (a path) connecting the two, but for the path to be active, every pair of two consecutive edges on that path must follow one of three patterns [11]:

$\ldots \rightarrow C \rightarrow \ldots \quad$ where $C$ is not in $X$

$\ldots \leftarrow C \rightarrow \ldots \quad$ where $C$ is not in $X$

$\ldots \rightarrow C \leftarrow \ldots$ where $C$ or a descendant of $C$ is in $X$.

In these patterns $C$ is the vertex between the two edges. If there is no active path between $A$ and $B$, then we say $X$ "d-separates" $A$ and $B$ (written $A \perp B \mid X$ ). The last case, in which the two edges point to the same vertex, is called a v-structure, and unless the two vertices that both point to $C$ share an edge (in which case we have a so-called moralized v-structure), conditioning on $C$ or a descendant of $C$ (a vertex with a directed path from $C$ ) allows information to flow through the path segment [10]. 


\section{OUTCOME MODELING}

In this paper, we assume that some existing model (the old model) has consistently been used to select samples for a database. We want to create a mathematical model (the new model) with this database that will hopefully better predict the outcome, and therefore be a better tool for future use. The old model can be either a formal selection model, such as a logistic regression on a feature vector, or informal, such as an interview by a loan agent. Both cases are discussed in later sections.

Bayesian networks are used to represent the relationship between the selection and outcome processes. As previously stated, the structure of the network depends on our assumptions about conditional independence, but the random variables corresponding to the vertices of the different graphs will be the same in all cases.

Following are the definition of each variable, with an example in parentheses of what the variable would represent in the case of loan applications.

- $y$ is the outcome variable. If $y$ is binary, $y=1$ if the outcome was good (loan repaid; the applicant was a good borrower), otherwise $y=0$.

- $s$ is the selection variable (indicating whether a loan application was accepted). If $s$ is binary, $s=1$ when the data point is selected for observation and $y$ is observable, otherwise $s=0$ and we cannot observe $y$.

- $x_{1}$ is a set of observed variables, also called covariates or features, available for training the new model (credit history, income level, age, etc.)

- $x_{2}$ is a set of unobserved variables (for example, unquantified traits such as an interviewer's general impression of the applicant's responsibility). These might have been used in the old model to help with selection, and might be variables influencing the outcome, but they are not available for training a new model.

- $x$ is sometimes used to mean $x_{1} \cup x_{2}$. This can be done in a Bayesian network without loss of generality by grouping the $x_{1}$ and $x_{2}$ vertices together into an $x$ vertex, which corresponds to not factoring the $p\left(x_{1}, x_{2}, \ldots \mid \ldots\right)=p\left(x_{1}, \ldots \mid x_{2} \ldots\right) p\left(x_{2}, \ldots \mid \ldots\right)$ term of the joint probability.

Our learning task can always be described by the following Bayesian network, since its skeleton is a complete graph and therefore the implied factoring of the joint distribution is completely general:

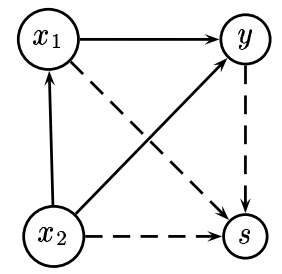

Template for alternative reject inference scenarios.

This network is a template for the different cases considered in this paper; all of the conditional independence relationships in the next section will follow from subgraphs of this graph, where we require the solid edges to be present and the dashed edges are optional. This results in eight different sets of assumptions, the first of which has no optional edges and the last of which has all the optional edges.

These constraints restrict the set of Bayesian networks to those with valid semantic interpretations (in terms of causality), even though those that lack valid causal interpretations may lead to equally useful statistical models. In general the $x$-variables are causes, and the outcome and selection variables, $y$ and $s$, are effects, so there should never be an edge from $y$ or $s$ to an $x$ variable. In the case of the selection procedure, this constraint conforms to what is done in practice: data points are selected for observation depending on features of that data point. In the case of the outcome event, this constraint conforms to our intuitive notions about causality: the outcome for a data point is influenced by the features of that data point.

We always assume the outcome, $y$, is dependent both on the observable variables $x_{1}$ and on the unobservable variables $x_{2}$. The edge from $x_{2}$ to $x_{1}$ conforms to our intuitive notions because observable variables, such as one's credit history, are influenced by unobservable variables, such as one's responsibility. This also helps in practice because it is often the case that the variables used in an old model are not present to train the new model. For example if an interview is part of the loan application process, the interviewer's general impression of the applicant can affect the decision to select the applicant, but it is not recorded in a database and will not be present for learning a new model.

In most cases there should be no edge between $y$ and $s$. Even though there can be a direct influence of $s$ on $y$, for example if $s$ represents selecting someone for treatment and $y$ represents survival, we do not add this edge into the graph. In this paper we are not trying to measure the probability of an outcome conditioned on whether or not an individual is selected, but instead to obtain an unbiased model of what the outcome would be for any individual in the general population, if selected. It is important to distinguish [3] between modeling the average risk difference, which is a function of $P$ (outcome|treatment) and $P$ (outcome|no treatment), and modeling only $P$ (outcome|treatment), which is the focus of this paper.

The reject inference problem can now be defined more formally. Note that it is actually a learning problem, not an inference (i.e. reasoning) problem. Our data set contains samples drawn independently at random from some underlying distribution, $p\left(x_{1}, x_{2}, y, s\right)$, where each sample for which $s=0$ is missing the value of $y$. The goal is to model the distribution of the outcome $y$ as a function of the observable variables $x_{1}$, i.e. to learn $p\left(y \mid x_{1}\right)$.

\section{CASES OF CONDITIONAL INDEPEN- DENCE}

The subsections in this section describe different possible independence relationships between the four variables, and how these relationships change the reject inference problem. Each set of conditional independence relationships follows from a Bayesian network that is a subgraph of the template in Section 3. Also provided are real-world situations in which these cases could arise. Algorithms for learning under these different assumptions are given in Section 5 . 


\subsection{Random selection}

In this case the following independence relationship holds:

$$
s \perp y, x_{1}, x_{2}
$$

which implies

$$
\begin{aligned}
& p\left(s \mid y, x_{1}, x_{2}\right)=p(s) \\
& p\left(y, x_{1}, x_{2} \mid s\right)=p\left(y, x_{1}, x_{2}\right) .
\end{aligned}
$$

This can be represented by a Bayesian network in which $s$ is not connected to any other random variables.

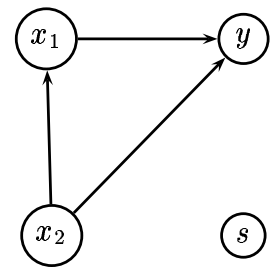

Case 1.

This is the most general subgraph (of the template) satisfying the independence relationships because any probability distribution with the given independence relationships will factor over it; a proper subgraph $G$ of this graph will certainly have the given independence relationship, but a probability distribution in which the marginal $p\left(x_{1}, x_{2}, y\right)$ cannot be further factored will not factor over $G$ but will factor over the graph of case 1 .

Factoring the joint probability of $x_{1}, x_{2}, y$, and $s$ once gives

$$
p\left(x_{1}, x_{2}, y, s\right)=p\left(x_{1}, x_{2}, y \mid s\right) p(s) .
$$

We find the marginal distribution $p\left(x_{1}, x_{2}, y\right)$ by summing over $s$ :

$$
\begin{aligned}
& p\left(x_{1}, x_{2}, y\right)=\sum_{s} p\left(x_{1}, x_{2}, y, s\right) \\
& \quad=p\left(x_{1}, x_{2}, y \mid s=1\right) p(s=1)+p\left(x_{1}, x_{2}, y \mid s=0\right) p(s=0) .
\end{aligned}
$$

Since $s$ is independent of all other variables, we have

$$
p\left(x_{1}, x_{2}, y \mid s=0\right)=p\left(x_{1}, x_{2}, y \mid s=1\right) .
$$

Making this substitution,

$$
\begin{aligned}
p\left(x_{1}, x_{2}, y\right)= & p\left(x_{1}, x_{2}, y \mid s=1\right) p(s=1) \\
& +p\left(x_{1}, x_{2}, y \mid s=1\right) p(s=0) \\
= & p\left(x_{1}, x_{2}, y \mid s=1\right)(p(s=1)+p(s=0)) \\
= & p\left(x_{1}, x_{2}, y \mid s=1\right)
\end{aligned}
$$

So the distribution of the selected samples, those for which $s=1$, is the same as the underlying distribution of $x_{1}, x_{2}$, and $y$. Any learner that only uses the selected data to learn this distribution will be learning an unbiased classifier.

This situation arises in randomized studies, in which samples are selected for observation completely at random. In the literature, this case is called missing completely at random (MCAR) [6].

\subsection{Selection conditionally independent of out- come}

In this case, we allow only the observable features to influence the selection. Thus the outcome and selection are conditionally independent:

$$
s \perp y \mid x_{1}
$$

which implies the constraints

$$
\begin{aligned}
p\left(s \mid x_{1}, y\right) & =p\left(s \mid x_{1}\right) \\
p\left(y \mid x_{1}, s_{1}\right) & =p\left(y \mid x_{1}\right) .
\end{aligned}
$$

This case is less restrictive than case 1 since any distribution satisfying the unconditional independence relationships of case 1 will also satisfy the conditional independence relationships of case 2. The most general Bayesian network that has this property and is a subgraph of the template is

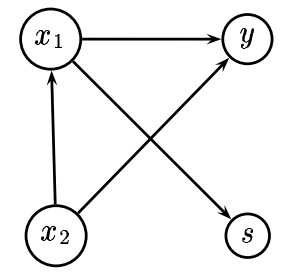

Case 2.

In this graph, observing $x_{1} \mathrm{~d}$-separates $y$ and $s$, so the conditional independence relationships are preserved. In this case, selection may depend on $x_{1}$, but given $x_{1}, y$ adds no additional information about selection, or, equivalently, given $x_{1}$, knowing $s$ gives no information about the outcome (for example, whether or not someone is a bad borrower).

This situation can arise if a formal selection model is used, where $s$ is some function of the observable variables, $x_{1}$. Rather confusingly, in the literature, this case is called missing at random (MAR) [6].

An important consequence of this conditional independence relationship is that the distribution of selected samples, $p\left(x_{1}, y \mid s=1\right)$, is related to the underlying distribution $p\left(x_{1}, y\right)$ in the following way:

Lemma 1: Under case 2, $p\left(x_{1}, y\right)=\frac{p(s=1)}{p\left(s=1 \mid x_{1}\right)} p\left(x_{1}, y \mid s=1\right)$ if all three probabilities are non-zero.

Proof: Apply Bayes' rule to the right hand side of this equation and then use the assumed conditional independence relationship:

$$
\begin{aligned}
& \frac{p(s=1)}{p\left(s=1 \mid x_{1}\right)} p\left(x_{1}, y \mid s=1\right) \\
& =\frac{p(s=1)}{p\left(s=1 \mid x_{1}\right)} \frac{p\left(s=1 \mid x_{1}, y\right) p\left(x_{1}, y\right)}{p(s=1)} \\
& =\frac{p(s=1)}{p\left(s=1 \mid x_{1}\right)} \frac{p\left(s=1 \mid x_{1}\right) p\left(x_{1}, y\right)}{p(s=1)} \\
& =p\left(x_{1}, y\right) \text {. }
\end{aligned}
$$

Lemma 1 is applied in Section 5.2 to learn an unbiased model in this case. Sample weights are created to rebalance the training set so that the weighted distribution of the selected samples is the same as the (unweighted) distribution of the general population [16].

\subsection{Selection dependent on outcome}

In this case, the selection is dependent on the outcome, but given the outcome, selection is not dependent on the covariates, observed or unobserved:

$$
s \perp x \mid y
$$

or in other words

$$
\begin{aligned}
& p(s \mid x, y)=p(s \mid y) \\
& p(x \mid s, y)=p(x \mid y)
\end{aligned}
$$


The most general subgraph that has this property is

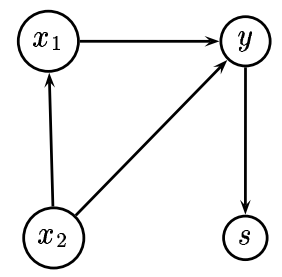

Case 3 .

The important feature of this graph is that $y$ d-separates $s$ and $x$.

If the variables $s$ and $y$ are binary, this situation corresponds to differences in the base rates of $y$ for the selected data and the general population. This situation arises most often when the record-keeping procedure deletes outcome labels depending on the outcome label (also known as censoring). An example in medical studies is estimating expected survival time from a database in which patients who lived longer than five years do not have a survival time recorded. Section 5.3 discusses what may and may not be learned in this case.

This situation also arises when the biased selection changes the distribution of $y$, but the conditional distributions of $x$ given each possible outcome $y$ are unchanged, because in the network $y$ d-separates $x$ and $s$. For example, suppose we learn to diagnose a disease $y$ based on patients $x$ encountered at one hospital. At a different hospital, the prevalence of the disease $p(y)$ may be different, but it is often reasonable to assume that the characteristics of affected and unaffected patients, i.e. $p(x \mid y=1)$ and $p(x \mid y=0)$, are unchanged. This scenario has been analyzed previously [5].

\subsection{Conditional independence with selection that can be modeled}

In this case, the only conditional independence relationship asserts that the unobserved covariates cannot influence selection:

$$
s \perp x_{2} \mid x_{1}, y
$$

or

$$
p\left(s \mid x_{1}, x_{2}, y\right)=p\left(s \mid x_{1}, y\right) .
$$

The most general graph that has this property is a combination of the previous two. This creates two possible vstructures, but both are moralized so there are no unconditional independence relationships due to the v-structures:

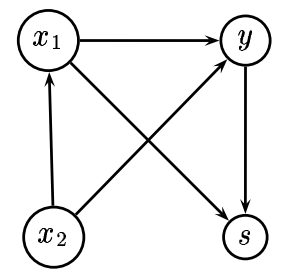

Case 4 .

This situation could arise in practice if a formal selection model is used to determine $s$, and then in addition $p(y)$ changes as discussed in the previous subsection. Learning under these assumptions is discussed in Section 5.4.

\subsection{Conditionally independent selection with missing features-selection that cannot be modeled}

This case is a generalization of case 2 in which the unobserved features in $x_{2}$ may influence selection. This occurs in practice when a selection decision is made based on unrecorded information, such as an interviewer's general impression, or numerical features which are not available for learning the new model. The conditional independence relationship describing this case is

$$
s \perp y \mid x_{1}, x_{2} .
$$

However, $x_{2}$ can never be observed, so it is impossible to make use of any independence relationships.

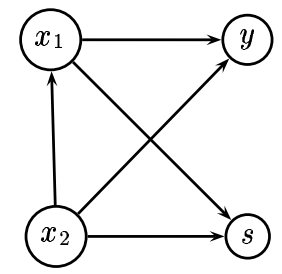

Case 5 .

\subsection{Outcome conditionally independent selec- tion with only missing features-unlearn- able selection}

This case is a special case of the previous case. All the features that influence the selection variable are in $x_{2}$ and not $x_{1}$ :

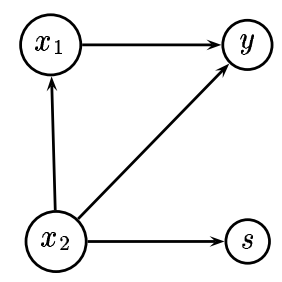

Case 6.

In this case it is impossible to learn a model of the selection mechanism. This case rarely arises. Intuitively, if the selection mechanism and the outcome mechanism are related (as they are in loan applications; loan approval attempts to predict loan repayment) and the outcome depends in some way on the available features in $x_{1}$, then it is reasonable to expect the selection to depend in some way on $x_{1}$.

This case could arise if a lending institution is just beginning to use statistical methods in its loan application procedure. Such an institution would have a training set consisting entirely of people whose loan applications were approved solely on the basis of an agent's subjective judgment, and not recordable features.

\subsection{Selection that cannot be modeled}

This cases features a selection mechanism that is not influenced by the observable features, $x_{1}$, but is influenced by 
unobservable features $x_{2}$ and by the outcome $y$ :

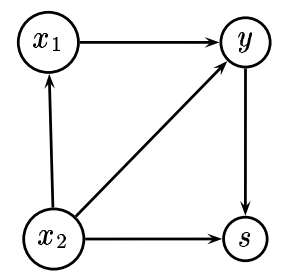

Case 7.

This scenario arises if the predictive features of the selection mechanism are not present for training a new model, and in addition outcome labels are missing in a way that depends on the value of the outcome.

\subsection{Arbitrarily biased selection}

This case is the most general: there are no assumptions about conditional independence relationships. The Bayesian network representing this case is

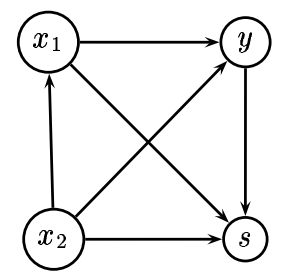

Case 8.

Since this Bayesian network represents a completely general factoring of the joint distribution, we would be able to use it to model a situation in which any of the previous conditions hold. This model should be used in practice when nothing is known about the selection mechanism.

\section{REJECT INFERENCE ALGORITHMS}

As stated above, Bayesian networks have no capability to encode the relationship between the outcome and selection variables, so our algorithms must learn from a heterogeneous training set, one in which there exist two different schemas for data in the training set.

\subsection{Learning under case 1}

Since the distribution $p\left(y, x_{1}, x_{2} \mid s=1\right)$ is the same as the underlying distribution $p\left(y, x_{1}, x_{2}\right)$, any classifier that learns from only the selected samples will learn an unbiased model of the outcome. However, because this classifier is based on fewer data points, it will have increased variance.

\subsection{Learning under case 2}

When we want to predict $y$ based on $x$, we usually have the choice of learning $p(y \mid x)$ or learning $p(x, y)$. The former is often called discriminative learning, while the latter is called learning a generative model. Under case 2, these two types of learning are very different.

\section{Discriminative modeling}

Given any particular $x$, the distribution of $y$ for the selected data is the same as the distribution of $y$ for the unselected data, because

$$
p\left(y \mid x_{1}, s=0\right)=p\left(y \mid x_{1}\right)=p\left(y \mid x_{1}, s=1\right) .
$$

So learning a model of the outcome based only on selected data provides an unbiased estimate of $p(y \mid x)$ in the entire population.

Intuitively the samples with unobserved outcomes can contribute nothing to the model, since the values of the variable whose density we are trying to estimate are missing. This can be shown formally by looking at the contribution of each sample to the likelihood of the parameters of a model.

Assuming samples are drawn independently, the likelihood of the parameters given the data equals the product of the probabilities of every sample, given those parameters. If a value is missing in a particular sample, the probability of that sample equals the full joint distribution marginalized with respect to the missing feature. For example if the probability of some sample $i$ should be $p\left(A=a_{i}, B=b_{i}, C=\right.$ $\left.c_{i} \mid \Theta\right)$ but the value of $B$ is missing, the probability becomes $\sum_{b} p\left(A=a_{i}, B=b, C=c_{i} \mid \Theta\right)[1]$. It may seem counterintuitive that the likelihood increases if features are missing; however, if we interpret the likelihood as the goodness-of-fit of our model, then with less information (due to missing features), there is less opportunity to criticize our model and the likelihood should therefore be greater.

Rejected samples are missing the value of $y$, so $y$ should be treated as a missing feature in the conditional likelihood equation:

$$
L(\Theta \mid X)=\prod_{j=1}^{n} L_{j}(\Theta)
$$

where

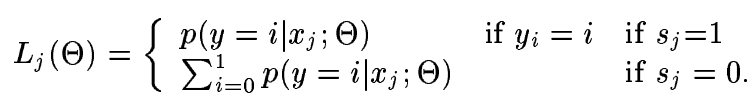

The sum equals 1 because $p(y=1)=1-p(y=0)$, so the contribution to the likelihood of the unselected data is an uninformative factor of 1 .

\section{Generative modeling}

If instead of modeling $p(y \mid x)$ we want to model $p(x, y)$, without loss of generality we can use a mixture model with one mixture component for the data in which $y=1$ and one mixture component for the data in which $y=0$. These mixture components come from the factoring $p(x, y)=p(y) p(x \mid y)$, which can be written in the case of binary $y$ as

$$
p(x, y)=p(y=0) p(x \mid y=0)+p(y=1) p(x \mid y=1) .
$$

The factors $p(y=i)$ are the mixing proportions, renamed $\pi_{i}$, and the conditional distributions $p(x \mid y=i)$ are modeled by the mixture components, written $p(x \mid y=i)=p_{i}(x)$. Now all samples are modeled as being drawn from the two component mixture

$$
p(x \mid y)=\pi_{o} p_{0}(x)+\pi_{1} p_{1}(x) .
$$

We only observe which mixture component generated a sample for those samples with observed outcomes. The contribution to the likelihood for the observed samples is

$$
p\left(x_{i}, y_{i}\right)=p\left(y_{i}\right) p\left(x_{i} \mid y=y_{i}\right)=\pi_{y_{i}} p_{y_{i}}\left(x_{i}\right) .
$$

As before, the contribution to the likelihood for those samples with an unobserved outcome is obtained by marginalizing with respect to the outcome variable:

$$
p\left(x_{i}, y_{\text {missing }}\right)=\sum_{y} p\left(x_{i}, y\right)=\sum_{i=0}^{1} \pi_{i} p_{i}(x) .
$$


Assuming there are $m$ rejected samples and $n$ accepted samples, the full likelihood of all data is

$$
L=\prod_{j=1}^{m}\left\{\sum_{i=0}^{1} \pi_{i} p_{i}\left(x_{j} \mid \theta_{i}\right)\right\} \prod_{j=m+1}^{m+n}\left\{\sum_{i=0}^{1} z_{i j} \pi_{i} p_{i}\left(x_{j} \mid \theta_{i}\right)\right\}
$$

where the indicator $z_{i j}$ equals 1 if $y_{j}=i$ and 0 otherwise. The log likelihood is

$$
\begin{aligned}
\log (L)= & \sum_{j=1}^{m} \log \left\{\sum_{i=0}^{1} \pi_{i} p_{i}\left(x_{j} \mid \theta_{i}\right)\right\} \\
& +\sum_{j=m+1}^{m+n} \sum_{i=0}^{1} z_{i j} \log \pi_{i} p_{i}\left(x_{j} \mid \theta_{i}\right)
\end{aligned}
$$

This equation contains a logarithm of sums for the unobserved samples, which makes an analytic maximum likelihood approach intractable. The EM algorithm is a common algorithmic approach to this maximization. EM iteratively optimizes the so-called complete-data log likelihood

$$
\log L\left(\Theta^{(t)}\right)=\sum_{j=1}^{m+n} \sum_{i=0}^{1} z_{i j}^{(t)} \log \pi_{i} p_{i}\left(x_{j} \mid \theta_{i}\right)
$$

where $z_{i j}^{(t)}$ is the estimate during iteration $t$ of the probability that $y=i$ for sample $j$. First, the E-step uses some initial estimate $\Theta^{(0)}$ of the parameters, based only on observed data, to estimate the probabilities $z_{i j}^{(1)}$. These probabilities are then used in the M-step to learn a new set of parameters, $\Theta^{(1)}$, that maximizes the likelihood of the data, including $z_{i j}^{(1)}$. The $\mathrm{E}$ and $\mathrm{M}$ steps are iterated until convergence [6].

\section{Reweighting, stratification, and propensity scoring}

Reweighting methods used to solve the reject inference problem, sometimes called inverse probability of treatment weighting (IPTW), weight each selected sample so that the distribution of weighted samples equals the underlying distribution ignoring selection [14]. These methods take advantage of Lemma 1.

The IPTW method first learns a select/reject model using the entire data set. After the model is adjusted to yield well-calibrated probabilities, sampling weights are created for each selected sample: $P(s=1) / P(s=1 \mid x)$. A model for $P\left(y \mid x_{1}\right)$ is then learned using the weighted (selected) samples, ignoring the unselected samples [16].

Stratified analysis (also called banded analysis) is a special case of reweighting. Like the IPTW method, the rejected samples are used only to create weights for the accepted samples. The stratified analysis presented in [4] is an approximation of the IPTW method. First an accept/reject model is estimated using the entire data set. The range of the classifier's output is partitioned into several strata or bands, usually so they contain similar numbers of samples. Within band $j$, the probability of selection is estimated as the ratio of selected samples in band $j$ to the total number of samples in band $j$. This essentially bins the classifier to estimate the conditional probability of selection given $x_{1}$. For a more adaptive binning method, see [15], which also divides the range of the classifier score into several bands (typically many more than stratified analysis) and assigns a probability to each band, estimated from the samples in that band and its neighbors. This calibration algorithm simultaneously determines an appropriate population size for each band and enforces the monotonicity of the probability given the score.

Within each band $j$, all of the accepted samples are given a weight of $1 / p(s=1 \mid j)$, which is an estimate of $1 / p(s=$ $1 \mid x)$. Here $j$ is a function of $x$ defined by a range of scores determined by the accept/reject model. This is the same weight used for the IPTW method, except for the constant factor $p(s=1)$. Since resampling is done with probability proportional to each sample's weight, and is not sensitive to constant factors, stratified analysis is equivalent to the IPTW method.

The propensity score method is a technique similar to stratified analysis, except that it is usually used to find an unbiased estimate of the average effect of some treatment on the general population, a function of $p$ (y|treatment) and $p(y \mid$ no treatment $)$, as opposed to solving the reject inference problem, which is to learn a per-individual model of the effect of treatment.

The propensity score method is described here to illustrate the similarities to stratified analysis. The propensity score is the function $e(x)=p(s=1 \mid x)$, the conditional probability of selection given $x$. It is a special case of the more general balancing score, any function $b(x)$ such that $x \perp s \mid b(x)$. It is shown in [13] that if selection and outcome are conditionally independent given $x$ (i.e. case 2 applies), then $y \perp s \mid e(x)$; for any particular value of $e(x)$, the distribution of the outcome variable is independent from the selection variable. Accepted samples are grouped (banded) together according to their propensity score [13]. Since the treatment effect (outcome) and selection are conditionally independent within one band, the treated population and the untreated population within the same band may be directly compared. An unbiased estimate of the average treatment effect is the average of the average treatment effects in each band, weighted by the number of samples in that band.

When the outcome is continuous, for example, the increase in white blood cells, the pair matching method may be used. When one treated sample and one untreated sample both have the same propensity score $e(x)$, the two are called a matched pair and the difference in outcome values is an unbiased estimate of the outcome difference at $e(x)$ [13].

\subsection{Learning under cases 3 and 4}

In case 3 , selection is independent of the covariates, both observed and unobserved, given the outcome: $s \perp x \mid y$. The bias only depends on the outcome label. Therefore the only difference between the distribution of selected samples and the distribution of the general population is a different prior for the outcome probability.

Without making further assumptions, it is impossible to infer the true baseline probability of a specific outcome in the general population, as can be seen by first applying Bayes' rule and then using the conditional independence relationship:

$$
\begin{aligned}
p\left(y=1 \mid x_{1}\right) & =\frac{p\left(x_{1} \mid y=1\right) p(y=1)}{p\left(x_{1}\right)} \\
& =\frac{p\left(x_{1} \mid y=1, s=1\right) p(y=1)}{p\left(x_{1}\right)} .
\end{aligned}
$$

The quantity $p\left(x_{1} \mid y=1, s=1\right)$ can be estimated from only observed samples, as can $p\left(x_{1}\right)$, but it is impossible to 
estimate $p(y=1)$ since this could have been arbitrarily altered.

If we learn separate models of the covariates of the good outcomes $p\left(x_{1} \mid y=1\right)$ and of the covariates of the bad outcomes $p\left(x_{1} \mid y=0\right)$, from the selected samples, and we have outside information that $p(y=1)=p_{1}$, then we can create an unbiased model of $p\left(y=1 \mid x_{1}\right)$ using Bayes' Rule:

$$
p\left(y=1 \mid x_{1}\right)=p_{1} \frac{p\left(x_{1} \mid y=1\right)}{p\left(x_{1} \mid y=0\right)+p\left(x_{1} \mid y=1\right)} .
$$

For further discussion of this case, see [5].

Under case 4 , the selection variable depends both on the observable features and the outcome variable. Without making further assumptions about the selection mechanism, it is impossible to learn an unbiased model to predict $y$, just as for case 3 .

\subsection{Learning under cases $5,6,7$, and 8}

In each of these cases, the selection mechanism can depend, at least in part, on unobservable features. This is especially relevant when correcting for self-selection bias, an example of which is estimating something about the general population from survey results when returning the surveys is voluntary (and therefore not random). The unobservable features prevent us from building an accurate model of the selection mechanism. However, even if we make no conditional independence assumptions we can still estimate an unbiased model of the outcome if we are willing to assume a particular functional form of the relationship between the outcome and selection processes. The usual approach given this new assumption is the bivariate probit, which assumes a normal distribution for the outcome and selection variables. Heckman's two-step estimator, an approximation of the maximum likelihood estimate of the bivariate probit parameters, is also explained below.

\section{Probit models}

The univariate probit model is used to represent the conditional distribution of a single response variable $y$ given a vector of features, based on a linear relationship between the features and a dummy variable $y^{*}$ which is not observed [2]:

$$
y_{i}^{*}=x_{i} \alpha+\epsilon .
$$

Here $x_{i}$ is the vector of features for sample $i, \alpha$ is a vector of parameters, and $\epsilon$ is a normally distributed random variable with zero mean and variance $\sigma^{2}$. The response $y_{i}$ for sample $i$ is related to $y_{i}^{*}$ by

$$
\begin{gathered}
y_{i}=1 \text { if } y_{i}^{*} \geq 0 \\
y_{i}=0 \text { if } y_{i}^{*}<0 .
\end{gathered}
$$

Given this linear relationship, we can write the functional form of $p(y=1 \mid x)$ :

$$
\begin{aligned}
p(y=1 \mid x) & =p\left(y^{*}>0 \mid x\right) \\
& =p(x \alpha+\epsilon>0) \\
& =p(\epsilon>-x \alpha) \\
& =1-p(\epsilon<-x \alpha) \\
& =1-\Phi_{\sigma}(-x \alpha) \\
& =\Phi_{\sigma}(x \alpha) \\
& =\Phi(x \alpha / \sigma) .
\end{aligned}
$$

Here $\Phi_{\sigma}($.$) is the cumulative normal distribution function$ with variance $\sigma^{2}$ and mean 0 , while $\Phi($.$) is the standard$ cumulative normal distribution function. Equation (2) is known as the probit equation. Given this functional form, the log likelihood of parameters $\Theta$ given data set $X$ with $n$ samples is

$\log L(\Theta \mid X)=\sum_{i=1}^{n} y_{i} \log \Phi\left(x_{i} \alpha / \sigma\right)+\left(1-y_{i}\right) \log \left(1-\Phi\left(x_{i} \alpha / \sigma\right)\right)$.

There are iterative methods for estimating $\alpha / \sigma$ given this model. Note that $\alpha$ and $\sigma$ are not uniquely defined since they only enter into the model as the ratio $\alpha / \sigma[2]$.

\section{Bivariate probit models}

Bivariate probit is an extension of univariate probit, in which there are two response variables (our outcome and selection variables) [6]. For all samples $i=1,2, \ldots, n$

$$
y_{i}^{*}=\mathbf{x}_{\mathbf{i}} \alpha+\epsilon_{\mathbf{i}}
$$

and

$$
s_{i}^{*}=\mathbf{x}_{\mathbf{i}} \beta+\nu_{\mathbf{i}} .
$$

Here $s_{i}^{*}$ and $y_{i}^{*}$ are unobserved variables. Analogously to the univariate probit, let the binary $s$ and $y$ equal 1 if the associated continuous variable is nonnegative, or 0 if it is negative. The vectors $\alpha$ and $\beta$ are the coefficients of the linear models, while $\epsilon_{i}$ and $\nu_{i}$ are the errors of those models for each sample, with variances $\sigma_{1}$ and $\sigma_{2}$ respectively.

We assert the selection rule: for sample $i, y_{i}$ is only observed if $s_{i}=1$. Assume the errors are bivariate normally distributed:

$$
\left(\begin{array}{c}
\epsilon_{i} \\
\nu_{i}
\end{array}\right) \sim N\left(\mu, \Sigma^{2}\right), \mu=\left(\begin{array}{c}
0 \\
0
\end{array}\right), \Sigma^{2}=\left(\begin{array}{cc}
\sigma_{1}^{2} & \sigma_{12} \\
\sigma_{12} & \sigma_{2}^{2}
\end{array}\right)
$$

Each of the three possible types of observation has a different form for its contribution to the likelihood:

$$
\begin{gathered}
s=0: P(s=0)=1-\Phi\left(x_{i} \beta / \sigma_{2}\right) \\
s=1, y=0: P(s=1, y=0)= \\
\Phi\left(x_{i} \beta / \sigma_{2}\right)-\Phi_{\rho}\left(x_{i} \alpha / \sigma_{1}, x_{i} \beta / \sigma_{2}\right) \\
s=1, y=1: P(s=1, y=1)=\Phi_{\rho}\left(x_{i} \alpha / \sigma_{1}, x_{i} \beta / \sigma_{2}\right)
\end{gathered}
$$

Here $\Phi$ is the standard univariate normal cumulative distribution function, as above, and $\Phi_{\rho}$ is the standard bivariate normal cumulative distribution with correlation $\rho=\frac{\sigma_{12}}{\sigma_{1} \sigma_{2}}$. Equation (3) holds by the definition of the probit model, $p(s=1)=\Phi\left(x_{i} \alpha / \sigma_{1}\right)$, and the fact that $p(s=0)=$ $1-p(s=1)$. Similar reasoning yields Equation (5). Equation (4) holds because

$$
\begin{aligned}
p(s=1, y=0) & =p(y=0 \mid s=1) p(s=1) \\
& =(1-p(y=1 \mid s=1)) p(s=1) \\
& =\left(1-\frac{p(y=1, s=1)}{p(s=1)}\right) p(s=1) \\
& =p(s=1)-p(y=1, s=1)
\end{aligned}
$$

and applying the probit model equations yields (4). These three expressions can combined into one log likelihood func- 
tion:

$$
\begin{array}{ll}
\log L\left(\alpha / \sigma_{1}, \beta / \sigma_{2}, \rho \mid X\right)= \\
\sum_{i=1}^{n} \quad\left(1-s_{i}\right) \log \left(1-\Phi\left(x_{i} \alpha / \sigma_{1}\right)\right) \\
\quad+s_{i}\left(1-y_{i}\right) \log \left(\Phi\left(x_{i} \alpha / \sigma_{1}\right)-\Phi_{\rho}\left(x_{i} \alpha / \sigma_{1}, x_{i} \beta / \sigma_{2}\right)\right) \\
\quad+s_{i} y_{i} \log \Phi_{\rho}\left(x_{i} \alpha / \sigma_{1}, x_{i} \beta / \sigma_{2}\right)
\end{array}
$$

From this equation we can derive iterative maximum likelihood estimators of $\alpha / \sigma_{1}, \beta / \sigma_{2}$, and $\rho$. The correlation $\rho$ between the errors of the two linear models, $\epsilon$ and $\nu$, indicates how and if the samples were selected in a biased way. If $\rho$ is zero there is no correlation, and selection is independent from the outcome given the observable features, so we can use one of the methods for case 2 to estimate an unbiased model. If $\rho$ is positive, then the unobserved features do affect selection and the outcome in a way that makes them positively correlated. Similarly, if $\rho$ is negative, then selection is an indicator of a bad outcome [6].

The maximum likelihood estimation of the outcome given the features is then $p(y=1 \mid x)=\Phi\left(x \alpha / \sigma_{1}\right)$, which is the probit equation (2). This estimate is asymptotically unbiased in theory, with its performance in practice depending on the correctness of the parametric assumption stated in equation (1).

\section{The two step Heckman estimator}

The computation of the model identified by the bivariate probit maximum likelihood equation was until recently prohibitively computationally intensive [12]. For this reason, Heckman developed a two step estimator to solve the reject inference problem when the outcome $y$ is continuous [7]. Many problems with binary outcomes have a natural correspondence with problems with a continuous outcome. For example, the problem of estimating the probability of survival, a problem with a binary outcome, can be extended to finding an unbiased estimate of the expected survival time. In the domain of credit scoring, the problem of estimating the probability of loan repayment can be cast as estimating how profitable a customer will be to the lending institution.

For consistency of notation, the outcome of sample $i$, now continuous, is denoted $y_{i}^{*}$ and is observed in samples for which $s_{i}=1$. The Heckman estimator relies on a property of the conditional distribution of $y_{i}^{*}$ given that $s_{i}^{*}>0$ (i. e. given $s_{i}=1$, the sample was selected). From [2],

$$
\begin{aligned}
E\left(y_{i}^{*} \mid s_{i}^{*}>0, x\right) & =E\left(y_{i}^{*} \mid \nu_{i}>-x_{i} \beta\right) \\
& =x_{i} \alpha+\frac{\sigma_{12}}{\sigma_{2}} \frac{\phi\left(-x_{i} \beta / \sigma_{2}\right)}{1-\Phi\left(-x_{i} \beta / \sigma_{2}\right)}
\end{aligned}
$$

where $\phi$ is the standard normal density function. The last term is usually expressed in terms of the function $\lambda()$, known as the inverse Mills ratio:

$$
\begin{aligned}
\lambda\left(Z_{i}\right) & =\frac{\phi\left(Z_{i}\right)}{1-\Phi\left(Z_{i}\right)} \\
Z_{i} & =-\frac{x_{i} \beta}{\sigma_{2}} .
\end{aligned}
$$

The two step Heckman procedure is as follows [9]:

1. Estimate the parameters $\beta / \sigma_{2}$ describing the probability of selection given $x$. This is a simple univariate probit as in equation (2), and the maximum likelihood solution can be found by iterative methods. For each sample, $Z_{i}$ can be estimated, and therefore the inverse Mills ratio can be estimated as $\hat{\lambda}_{i}=\lambda\left(Z_{i}\right)$.

2. Equation (6) becomes the linear regression

$$
E\left(y_{i}^{*} \mid s_{i}=1, x\right)=x_{i} \alpha+\sigma_{12} / \sigma_{2} \hat{\lambda}_{i}
$$

using $x_{i}$ and $\hat{\lambda}_{i}$ as the regressors. Solve this regression using ordinary least squares to estimate $\alpha$ and $\sigma_{12} / \sigma_{2}$.

From the second step, we get an estimate of $\sigma_{12} / \sigma_{2}$. Call this $C$. Let $\hat{V}_{i}^{2}$ be the squared residuals from the regression in the second step, and let $I_{1}$ be the number of samples for which $s=1$. Now we can estimate $\sigma_{1}$ as

$$
\hat{\sigma}_{1}^{2}=\frac{\sum_{i=1}^{I_{1}} \hat{V}_{i}^{2}}{I_{1}}-\frac{C}{I_{1}} \sum_{i=1}^{I_{1}}\left(\hat{\lambda}_{i} Z_{i}-\hat{\lambda}_{i}^{2}\right)
$$

Analogously to the bivariate probit model, the unbiased estimated model of the outcome given the features is $E\left(y^{*} \mid x\right)=$ $x \alpha$.

An interesting similarity between the reweighting methods of Section 5.2 and the Heckman procedure is that both first learn a model of the selection mechanism and then use that model to learn a model of the outcome mechanism.

\section{CONCLUSIONS AND FUTURE WORK}

The main contribution of this paper is to apply Bayesian networks to describe the different assumptions one may make in the course of developing a procedure to learn an unbiased model from a training set with sample selection bias (i.e. in the course of solving the reject inference problem). Our framework describes previously published cases as well as several novel ones. The use of Bayesian networks makes recognizing which case is applicable easier and more intuitive, since conditional independence relationships are easy to spot in the simple networks involved in reject inference.

For each case, we have provided an overview of the probabilistic learning algorithms that are available, to the extent to which a model can be learned in each case. In some cases a provably unbiased model can be learned, while in other case selection bias is impossible to overcome. In the most general case, we can only learn an unbiased model if a particular functional form for the outcome and selection models is assumed.

Future work includes investigating what algorithms exist to learn the parameters of Bayesian networks with a heterogeneous training set, i.e. a training set that contains examples with differing patterns of observed and unobserved variables.

None of the algorithms described above are specifically Bayesian network training algorithms [8]. An important direction for future work is to investigate how existing Bayesian network methods perform with a heterogeneous training set, i.e. a training set that contains examples with differing patterns of observed and unobserved variables. These algorithms can be used in principle to solve the reject inference problem. A related research direction is to apply structural learning algorithms to discover which of the cases in Section 4 best describes a given data set.

Alternatively, perhaps algorithms developed specifically to solve reject inference problems can be extended to learn the parameters of Bayesian networks given a heterogeneous training set. 


\section{ACKNOWLEDGMENTS}

The authors thank Fair Isaac Corporation for funding this research through California MICRO grant 2003-024. This research was also supported by a gift from Sun Microsystems.

\section{REFERENCES}

[1] P. D. Allison. Missing Data. Sage Publications, Inc., Thousand Oaks, California, 2002.

[2] T. Amemiya. Advanced Econometrics. Harvard University Press, Cambridge, Massachusetts, pages 267-359, 1985.

[3] D. A. Cobb-Clark and T. Crossley. Econometrics for evaluations: An introduction to recent developments. The Economic Record, 79(247):491-511, 2003.

[4] J. Crook and J. Banasik. Does reject inference really improve the performance of application scoring models? Technical Report Working Paper Series No. 02/3, Credit Research Centre, 2002.

[5] C. Elkan. The foundations of cost-sensitive learning. In $I J C A I$, pages 973-978, 2001.

[6] A. J. Feelders. An overview of model based reject inference for credit scoring. Technical report, Utrecht University, Institute for Information and Computing Sciences. http://www.cs.uu.nl/people/ad/mbrejinf.pdf.

[7] Y. Freund, H. Seung, E. Shamir, and N. Tishby. Information, Prediction, and Query by Committee. In Advances in Neural Information Processing Systems, vol. 5, pages 483-490, 1992.

[8] D. Heckerman. A Tutorial on Learning with Bayesian Networks. Technical report MSR-TR-95-06, Microsoft Research, (1995).

ftp://ftp.research.microsoft.com/pub/tr/tr-95-06.pdf.
[9] J. J. Heckman. Sample selection bias as a specification error. Econometrica, 47(1), pages 153-162, 1979.

[10] K. Murphy. Dynamic Bayesian Networks: Representation, Inference and Learning. $\mathrm{PhD}$ thesis, University of California, Berkeley, 2002.

[11] J. Pearl. Graphical models for probabilistic and causal reasoning. In D. M. Gabbay and P. Smets, editors, Handbook of Defeasible Reasoning and Uncertainty Management Systems, Volume 1: Quantified Representation of Uncertainty and Imprecision, pages 367-389. Kluwer Academic Publishers, Dordrecht, 1998.

[12] P. A. Puhani. The Heckman correction for sample selection bias and its critique. Journal of Economic Surveys, 14(1), 2000.

[13] P. R. Rosenbaum and D. B. Rubin. The central role of the propensity score in observational studies for causal effects. Biometrika, 70(1):41-56, 1983.

[14] B. Zadrozny. Learning and Evaluating Classifiers under Sample Selection Bias. Proceedings of the $21^{\text {st }}$ International Conference on Machine Learning, 2004.

[15] B. Zadrozny and C. Elkan. Transforming classifier scores into accurate multiclass probability estimates. Proceedings of the Eighth International Conference on Knowledge Discovery and Data Mining, pages 694-699, 2002.

[16] B. Zadrozny, J. Langford, and N. Abe. Cost-sensitive learning by cost-proportionate example weighting. IEEE International Conference on Data Mining (ICDM), pages 435-442, 2003. 